\title{
LIFTING OF TOPOLOGICAL ENTROPY
}

\author{
HARVEY B. KEYNES ${ }^{1}$
}

1. Introduction. In this paper, we will primarily be concerned with the following problem: Suppose the flow $(Y, \psi)$ is a transformation group homomorphic image of the flow $(X, \phi)$. What can be said about equality of the topological entropy of $(X, \phi)$ and the topological entropy of $(Y, \psi)$ ? In general, one can say nothing, since $(Y, \psi)$ may be the one-point flow. We show in this paper that group extensions and inverse limits do preserve entropy. This enables us to show that certain group-like extensions of minimal algebras (see [2]) also preserve entropy. In particular, it follows that minimal distal flows have zero entropy. We finally show that given $r \geqq 0$, there exists a flow whose entropy is $r$.

All spaces involved are compact Hausdorff. Unless otherwise stated, we will consider flows $(X, \phi)$, i.e., $\phi$ is a homeomorphism onto. However, Lemmas (2.2) thru (2.4), and Theorems (2.6) and (2.11) also hold for semiflows, i.e., continuous surjective maps. The entropy of $(X, \phi)$ will mean topological entropy and will be denoted by $h(X, \phi)$. All other notations and definitions for entropy will follow [1]. Finally, we will denote that $(Y, \psi)$ is a homomorphic image of $(X, \phi)$ by $(X, \phi) \stackrel{\sim}{\rightarrow}(Y, \psi)$.

\section{Lifting of entropy.}

(2.1). Definition (CF. [2], [3]). (a) The flow $(X, \phi)$ is an isometric extension of $(Y, \psi)$ if $\pi:(X, \phi) \stackrel{\rightarrow}{\rightarrow}(Y, \psi)$ and there exists a continuous map $\rho:\left\{\left(x, x_{1}\right) \mid \pi x=\pi x_{1}\right\} \rightarrow R$ such that:

(1) For every $y, \rho$ induces a metric on $\pi^{-1} y$.

(2) There exists a fixed compact metric space $M$ which is isometric to $\pi^{-1} y(y \in Y)$.

(3) If $x, x_{i} \in X$ and $\pi x=\pi x_{1}$, then $\rho\left(\phi^{n} x, \phi^{n} x_{1}\right)=\rho\left(x, x_{1}\right)$ for all $n$.

(b) The flow $(X, \phi)$ is a group extension of $(Y, \psi)$ if there exists a compact Hausdorff topological group $G$ such that:

(1) $(G, X)$ is a left freely-acting transformation group whose action commutes with $\phi$.

(2) The orbit transformation group $(X / G, \phi)$ is isomorphic to $(Y, \psi)$.

The following result is basically due to $\mathrm{R}$. Bowen.

Received by the editors September 9, 1968.

1 This work was supported by NSF Grant GP-8394. 
(2.2). Lemma. Let $K$ be a compact Hausdorff space, and $(X, \phi)$, $(Y, \psi)$ flows with $\pi:(X, \phi) \stackrel{\sim}{\rightarrow}(Y, \psi)$. Suppose that:

(a) For every $y \in Y$, there exists a homeomorphism $g_{y}$ of $K$ onto $\pi^{-1}(y)$ (with its subspace topology) such that given $\eta$ an index of $X$, there exists $\gamma$ an index of $K$ for which $g_{\nu} \times g_{\nu}(\gamma) \subset \eta(y \in Y)$.

(b) Given an index $\eta$ of $X$, there exists an index $\lambda$ of $X$ such that if $x, x_{1} \in X$ with $\pi x=\pi x_{1}$ and $\left(x, x_{1}\right) \in \lambda$, then $\left(\phi^{n} x, \phi^{n} x_{1}\right) \in \eta$ for all $n$. Then $h(X, \phi)=h(Y, \psi)$.

Proof. We need only show $h(X, \phi) \leqq h(Y, \psi)$. Let $\mathfrak{A}$ be a finite open cover for $X$, and $\eta$ a Lebesgue index for $\mathfrak{A}$. Choose an index $\gamma$ of $K$ as in (a), and an index $\lambda$ of $X$ as in (b). Then $\bigcup_{i=1}^{s} z_{i} \gamma=K$ for some integer $s$. By (b), $\cup_{i=1}^{s} g_{y}\left(z_{i}\right) \lambda=\pi^{-1} y(y \in Y)$. Applying (a), we have that $\phi^{j}\left(g_{y}\left(z_{i}\right) \lambda\right) \subset \phi^{j} g_{y}\left(z_{i}\right) \eta \subset U(y, j, i) \in \mathfrak{A}(y \in Y, j$ integer $\geqq 0,1 \leqq i \leqq s)$.

Fix an integer $m \geqq 0$. For every $y \in Y$, let $A(y, i)=\bigcap_{j=0}^{m-1} \phi^{-j} U(y, j, i)$ $(1 \leqq i \leqq s)$. Then $\bigcup_{i=1}^{s} A(y, i) \supset \pi^{-1}(y)$. Using the filter $\left\{\pi^{-1}(N) \mid N\right.$ a closed neighborhood of $y\}$, then $\bigcup_{i=1}^{s} A(y, i) \supset \pi^{-1}\left(M_{y}\right)$ for some open neighborhood $M_{\nu}$ of $y$. Choose a finite subset $F$ of $Y$ for which $\cup\left\{M_{y} \mid y \in F\right\}=Y$, and consider the open cover $\mathfrak{A}^{*}=\left\{M_{y} \mid y \in F\right\}$.

Now consider $\left(Y, \psi^{m}\right)$. Let $n$ be an integer $\geqq 0$, and $\mathfrak{A}_{n}^{*}$ a minimal

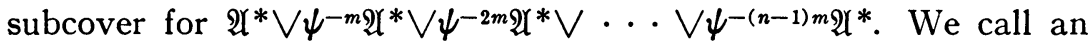
$n$-tuple $\left(y_{0}, \cdots, y_{n-1}\right) \in F^{n}$ admissible if $\bigcap_{i=0}^{n-1} \psi^{-i m} M_{y_{i}} \in \mathfrak{A}_{n}^{*}$. Choose $x \in X$ and let $y=\pi x$. Choose $\left(y_{0}, \cdots, y_{n-1}\right)$ admissible with $y \in \bigcap_{i=0}^{n-1} \psi^{-i m} M_{y_{i}}$. Then

$$
\begin{aligned}
x \in \pi^{-1}(y) \subset \bigcap_{i=0}^{n-1} \pi^{-1} \psi^{-i m} M_{y_{i}} & =\bigcap_{i=0}^{n-1} \phi^{-i m} \pi^{-1} M_{y_{i}} \subset \bigcap_{i=0}^{n-1} \phi^{-i m}\left(\bigcup_{k=1}^{s} A\left(y_{i}, k\right)\right) \\
& =\bigcap_{i=0}^{n-1} \bigcup_{k=1}^{s} \bigcap_{j=0}^{m-1} \phi^{-(i m+j)} U\left(y_{i}, j, k\right) \\
& =\bigcup_{\left(k_{0}, \cdots, k_{n}-1\right)} \bigcap_{i=0}^{n-1} \bigcap_{j=0}^{m-1} \phi^{-(i m+j)} U\left(y_{i}, j, k_{i}\right),
\end{aligned}
$$

where the first union is over $n$-tuples in $[1, s]^{n}$. Hence the cover $\mathfrak{A}_{n}=\left\{\bigcap_{i=0}^{n-1} \bigcap_{j=0}^{m-1} \phi^{-(i m+j)} U\left(y_{i}, j, \quad k_{i}\right) \mid\left(y_{0}, \cdots, y_{n-1}\right)\right.$ admissible, $\left.\left(k_{0}, \cdots, k_{n-1}\right) \in[1, s]^{n}\right\}$ is a subcover of $\mathfrak{A} \vee \phi^{-1 \mathfrak{A}} \bigvee \cdots \vee \phi^{-n m+1 \mathfrak{A}}$ such that $\left|\mathfrak{A}_{n}\right|=\left|\mathfrak{A}_{n}^{*}\right| s^{n}$, independent of $m$. Now

$1 / n m \cdot H\left(\mathfrak{A} \vee \cdots \vee \phi^{-n m+1} \mathfrak{U}\right)$

$$
\begin{aligned}
& \leqq H\left(\mathfrak{A}_{n}\right) / n m=\log \left(\left|\mathfrak{A}_{n}{ }^{*}\right| s^{n}\right) / n m \\
& =H\left(\mathfrak{A}^{*} \vee \cdots \vee \psi^{\left.-(n-1) m \mathfrak{Q} \mathfrak{I}^{*}\right) / n m}+\log s / m,\right.
\end{aligned}
$$

since $\mathfrak{A}_{n}^{*}$ is minimal. Letting $n \rightarrow \infty, h(\mathfrak{A}, \phi) \leqq h\left(\mathfrak{A}^{*}, \psi^{m}\right) / m+\log s / m$ 
$\leqq h\left(Y, \psi^{m}\right) / m+\log s / m=h(Y, \psi)+\log s / m$. Letting $m \rightarrow \infty, h(\mathfrak{A}, \phi)$ $\leqq h(Y, \psi)$. The result follows.

It is easy to see, using the continuity of $\rho$, that the metric topology on a fibre of an isometric extension is the subspace topology, whence (2.2) can be applied. We also have:

(2.3). Lemma. Let $(X, \phi)$ be a group extension of $(Y, \psi)$ with group $G$. Then $h(X, \phi)=h(Y, \psi)$.

Proof. In this case, each fibre is $G x$ for some $x \in X$. Since $G$ is freely acting, $\phi_{x}: G \rightarrow G_{x}, g \rightarrow g x$ is a homeomorphism $(x \in X)$, and a choice set of $X$ will give the homeomorphisms. As $G$ is a compact group of automorphisms of $(X, \phi)$ it follows by Ascoli's theorem and minimality properties of the compact-open topology that all the various function uniformities on $G$ will give the original uniformity. Applying this to the uniformity of uniform convergence on $G$ yields (a) of (2.2). For (b), we need only show that given an index $\eta$, there is an index $\gamma$ such that $(x, g x) \in \gamma$ for some $x$ implies $(z, g z) \in \eta$ for all $z \in X$. It is sufficient to show that given a neighborhood $U$ of $e$, there exists an index $\gamma$ of $X$ such that $(x, g x) \in \gamma$ for some $x$ implies $g \in U$. If not, there is some open neighborhood $V$ of $e$ such that given any index $\gamma$ of $X$, there exists $g_{\gamma} \notin V$ and $x_{\gamma} \in X$ for which $\left(x_{\gamma}, g_{\gamma} x_{\gamma}\right) \in \gamma$. By compactness, we can assume $x_{\gamma} \rightarrow x, g_{\gamma} \rightarrow g$. Then $g \notin V$. However $x=g x$, whence $G$ freely acting implies $g=e$. This is a contradiction. The result follows.

Note that if $(Z, \rho)$ is "in-between" $(Y, \psi)$ and a group extension $(X, \phi)$, then $h(Z, \rho)=h(Y, \psi)$.

(2.4). Lemma. Let $\left(\left(X_{\alpha}, \phi_{\alpha}\right) ; \pi_{\alpha}^{\beta}\right)$ be an inverse system of flows (i.e., if $\beta \geqq \alpha$, then $\left.\pi_{\alpha}^{\beta}:\left(X_{\beta}, \phi_{\beta}\right) \underset{\rightarrow}{\rightarrow}\left(X_{\alpha}, \phi_{\alpha}\right)\right)$. Let $(X, \phi)$ be the inverse limit flow. Then $h(X, \phi)=\sup h\left(X_{\alpha}, \phi_{\alpha}\right)$.

Proof. For every $\alpha$, let $\pi_{\alpha}:(X, \phi) \widetilde{\rightarrow}\left(X_{\alpha}, \phi_{\alpha}\right)$ be the induced canonical homomorphism. It then follows that $h(X, \phi) \geqq \sup h\left(X_{\alpha}, \phi_{\alpha}\right)$. Now let $\mathfrak{A}$ be an open cover for $X$. Since $\left\{\pi_{\alpha}^{-1}\left(U_{\alpha}\right) \mid U_{\alpha}\right.$ open in $X_{\alpha}$ open in $X_{\alpha}, \alpha$ arbitrary $\}$ is a base for $X$, it follows that $\mathfrak{A}$ is refined by an open cover $\mathfrak{B}$ with elements of the form $\pi_{\alpha}^{-1} U_{\alpha}$. By compactness, we can assume $\mathfrak{B}=\left\{\pi_{\alpha_{i}}^{-1}\left(U_{\alpha_{i}}\right) \mid i=1, \cdots, n\right\}$. Let $\alpha \geqq \alpha_{i}, i=1$, $\cdots, n$. Since $\pi_{\alpha_{i}}^{-1}\left(U_{\alpha_{i}}\right)=\pi_{\alpha}^{-1}\left(\left(\pi_{\alpha_{i}}^{\alpha}\right)^{-1}\left(U_{\alpha_{i}}\right)\right)(i=1, \cdots, n)$, we have that $\mathfrak{B}=\left\{\pi_{\alpha}^{-1}\left(V_{i}\right) \mid i=1, \cdots, n\right\}$, where $V_{i}$ open in $X_{\alpha}$. Thus, $\mathfrak{B}$ satisfies $\pi_{\alpha}^{-1} \pi_{\alpha} \mathfrak{B}=\mathfrak{B}$, and $\pi_{\alpha} \mathfrak{B}$ is an open cover of $X_{\alpha}$. Hence $h(\phi, \mathfrak{A})$ $\leqq h(\phi, \mathfrak{B})=h\left(\phi_{\alpha}, \pi_{\alpha} \mathfrak{B}\right) \leqq h\left(X_{\alpha}, \phi_{\alpha}\right) \leqq \sup h\left(X_{\alpha}, \phi_{\alpha}\right)$. Thus, $h(X, \phi)$ $\leqq \sup h\left(X_{\alpha}, \phi_{\alpha}\right)$, and the proof is completed.

As a corollary, if the inverse system $\left(\left(X_{\alpha}, \phi_{\alpha}\right) ; \pi_{\alpha}^{\beta}\right)$ with inverse 
limit flow $(X, \phi)$ satisfies $h\left(X_{\alpha}, \phi_{\alpha}\right)=c$ for every $\alpha$, then $h(X, \phi)=c$. Moreover, if the system is really a sequence, then $h(X, \phi)=$ $\lim h\left(X_{m}, \phi_{m}\right)$. We also have:

(2.5). Corollary. Let $\left(\left(X_{i}, \phi_{i}\right) \mid i \in I\right)$ be a family of flows. Then

$$
\begin{aligned}
h\left(X_{i} X_{i}, X_{i} \phi_{i}\right) & =\sup \left\{h\left(X_{F} X_{i}, X_{F} \phi_{i}\right) \mid F \text { a finite subset of } I\right\} \\
& \leqq \sup \left\{\sum_{F} h\left(X_{i}, \phi_{i}\right) \mid F \text { a finite subset of } I\right\} .
\end{aligned}
$$

Proof. The first equality follows from the fact that a product is an inverse system over finite subsets of the indexing set. The latter inequality comes from the obvious assertion that $h\left(\times_{F} X_{i}, X_{F} \phi_{i}\right)$ $\leqq \sum_{F} h\left(X_{i}, \phi_{i}\right)$ when $F$ is finite (whether equality holds here is, as yet, unknown).

Finally, the proof of (2.4) shows that if $\left(\left(X_{\alpha}, \phi_{\alpha}\right)\right)$ is a family of flows and $(X, \phi)$ is a flow such that

(1) there exists $\pi_{\alpha}:(X, \phi) \underset{\rightarrow}{\rightarrow}\left(X_{\alpha}, \phi_{\alpha}\right)$ for all $\alpha$, and

(2) every open cover of $X$ is refined by a cover of the form $\pi_{\alpha}^{-1} \mathfrak{A}_{\alpha}$, where $\mathfrak{A}_{\alpha}$ is an open cover of $X_{\alpha}$ for some $\alpha$, then $h(X, \phi)=\sup$ $h\left(X_{\alpha}, \phi_{\alpha}\right)$.

We now apply (2.3) and (2.4). The following result is immediate by (transfinite) induction.

(2.6). Theorem. Let $(X, \phi)$ be a flow for which there exist an ordinal $\nu$ and families $\left(\left(X_{\alpha}, \phi_{\alpha}\right) \mid \alpha \leqq \nu\right),\left(\left(Y_{\alpha}, \psi_{\alpha}\right) \mid \alpha \leqq \nu\right)$ of flows such that:

(1) $\left(X_{\nu}, \phi_{v}\right)=(X, \phi)$.

(2) If $\alpha<\nu,\left(Y_{\alpha+1}, \psi_{\alpha+1}\right)$ is a group extension of $\left(X_{\alpha}, \phi_{\alpha}\right)$.

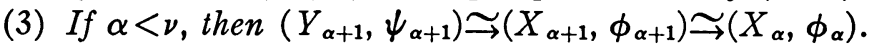

(4) If $\alpha \leqq \nu$ is a limit ordinal, then $\left(X_{\alpha}, \phi_{\alpha}\right)$ is the inverse flow of the inverse system $\left(\left(X_{\beta}, \phi_{\alpha}\right) \mid \beta<\alpha\right)$ defined by (3). Then $h(X, \phi)=h\left(X_{0}, \phi_{0}\right)$.

The principal application of (2.6) is to minimal flows. A careful reading of [2, Proposition 4.25$]$ yields:

(2.7). Proposition. Let $a$ and $B$ be T-subalgebras of $\mathfrak{N}$ such that $B$ is a nontrivial, quasi-separable, group-like extension of $a$. Then there exists an ordinal $\nu$ and collections $\left(B_{\alpha} \mid \alpha \leqq \nu\right),\left(\odot_{\alpha} \mid \alpha \leqq \nu\right)$ of $T$-subalgebras such that:

(1) $B_{0}=Q, B_{\nu}=@$.

(2) $\beta_{\alpha} \subset B_{\beta}(\alpha \leqq \beta \leqq \nu)$.

(3) $B_{\alpha+1}$ is an isometric extension of $B_{\alpha}$ and $\otimes_{\alpha+1}$ is a group extension sion of $B_{\alpha}$ with $B_{\alpha} \subset \bigotimes_{\alpha+1} \subset \mathcal{P}_{\alpha+1}(\alpha<\nu)$.

(4) For limit ordinals $\alpha \leqq \nu$,

$$
\Theta_{\alpha}=\left\{U\left\{\Theta_{\beta} \mid \beta<\alpha\right\}\right\}, \quad \odot_{\alpha}=\left\{U\left\{\odot_{\beta} \mid \beta<\alpha\right\}\right\} .
$$


Since $T$ is the integers in our case, every group-like extension is quasi-separable $[2$, p. 24]. The following result is then an immediate corollary of (2.6), noting that the limit algebras in (2.7) yield inverse limit transformation groups.

(2.8). Theorem. Let $(X, \phi)$ and $(Y, \psi)$ be minimal flows for which $X \sim \beta, Y \sim Q$, where $Q$ and $B$ are $T$-subalgebras of $\mathrm{T}$. Suppose that $B$ is a group-like extension of $\alpha$. Then $h(X, \phi)=h(Y, \psi)$.

(2.9). Corollary. Let $(X, \phi)$ be minimal distal. Then $h(X, \phi)=0$.

Proof. It is shown in [2] that a minimal distal flow is a group-like extension of the one-point flow.

Note that (2.9) for metric $X$ can be deduced directly from the Furstenberg Structure Theorem [3] by using (2.6).

We now construct flows with arbitrary entropy.

(2.10). Lemma. Let $(Y, \psi)$ be a flow and $n$ a positive integer. Form the flow $(X, \phi)$, where $X$ is $n$ disjoint copies $(Y, i)$ of $Y,(1 \leqq i \leqq n)$, and $\phi(y, i)=(y, i+1), 1 \leqq i \leqq n-1 ; \phi(y, n)=(\psi(y), 1)$. Then $h(X, \phi)$ $=n^{-1} h(Y, \psi)$.

Proof. It is clear that each $(Y, i)$ is $\phi^{n}$-invariant and that the flow $\left((Y, i), \phi^{n}\right)$ is isomorphic to $(Y, \psi)$. Hence $h\left((Y, i), \phi^{n}\right)=h(Y, \psi)$. Moreover, $h\left(X, \phi^{n}\right)=\max _{i} h\left((Y, i), \phi^{n}\right)=h(Y, \psi)$ by [1, Theorem 4$]$. Since $n h(X, \phi)=h\left(X, \phi^{n}\right)[1$, Theorem 2], the result follows.

(2.11). THEOREM. Let $r \geqq 0$. Then there exists a flow $(X, \phi)$ such that $h(X, \phi)=r$.

Proof. We can clearly assume $r>0$. Choose a flow $(Z, \rho)$ for which $h(Z, \rho)=c>0$ (e.g., a symbolic flow). If $m, n$ are positive integers, there exists a flow with entropy $m c / n$ by applying $(2.10)$ to $\left(Z, \rho^{m}\right)$. Pick an increasing sequence of rationals $\left(r_{n}\right)_{n \geqq 1}$ such that $r_{n} c \rightarrow r$, and let $\left(X_{n}, \phi_{n}\right)$ be a flow with entropy $r_{n} c$. Let $\left(X_{0}, \phi_{0}\right)$ be the trivial flow. Let $F$ be the finite subsets of the positive integers directed by subset inclusion. For every $F \in \mathcal{F}$, let $\left(X_{F}, \phi_{F}\right)$ be the disjoint sum flow $+\left(\left(X_{n}, \phi_{n}\right) \mid n \in F \cup\{0\}\right)$. If $F \leqq G$, define $\pi_{F}^{G}:\left(X_{G}, \phi_{G}\right) \stackrel{\sim}{\rightarrow}\left(X_{F}, \phi_{F}\right)$ by $\pi_{F}^{G} \mid X_{n}=$ id, if $n \in F$, and $\pi_{F}^{G} \mid X_{n}$ is the constant map onto $X_{0}$, if $n \notin F$. Let $(X, \phi)$ be the inverse limit flow of this system. Then $h(X, \phi)=\sup \left\{h\left(X_{F}, \phi_{F}\right) \mid F \in \mathcal{F}\right\}$ by (2.4). But $h\left(X_{F}, \phi_{F}\right)=\max _{F} r_{n} c$ since $\left(r_{n} c\right) \uparrow$. Thus, $h(X, \phi)=\sup _{n} r_{n} c=r$. The result follows.

It would be interesting to find a minimal flow which satisfies the conclusion of (2.11). It is known [4] that there exist minimal flows with arbitrarily large entropy. 
We finally consider a nonminimal distal flow $(X, \phi)$. Although $X$ decomposes into a partition of minimal distal flows, we cannot conclude that $h(X, \phi)=0$, since the partition may be infinite. We do know:

(2.12). Remark. Let $(X, \phi)$ be a distal flow and $(E(X), \psi)$ its enveloping semigroup. Then $h(E(X), \psi)=0$.

Proof. Since $(X, \phi)$ is distal, it is known that $(E(X), \psi)$ is minimal distal. The result follows by (2.8).

The author conjectures that if $(X, \phi)$ is a distal flow, then $h(X, \phi)$ $=0$.

\section{BIBLIOGRAPHY}

1. R. L. Alder, A. G. Konheim and M. H. McAndrew, Topological entropy, Trans. Amer. Math. Soc. 114 (1965), 309-319.

2. R. Ellis, The structure of group-like extensions of minimal sets, Trans. Amer. Math. Soc. 134 (1968), 261-287.

3. H. Furstenberg, The structure of distal flows, Amer. J. Math. 85 (1963), 477-515. MR $28 \# 602$.

4. F. Hahn and Y. Katznelson, On the entropy of uniquely ergodic transformations, Trans. Amer. Math. Soc. 126 (1967), 335-360. MR 37 \#7772.

University of California, Santa Barbara and

UNIVERSITY OF MiNNESOTA 